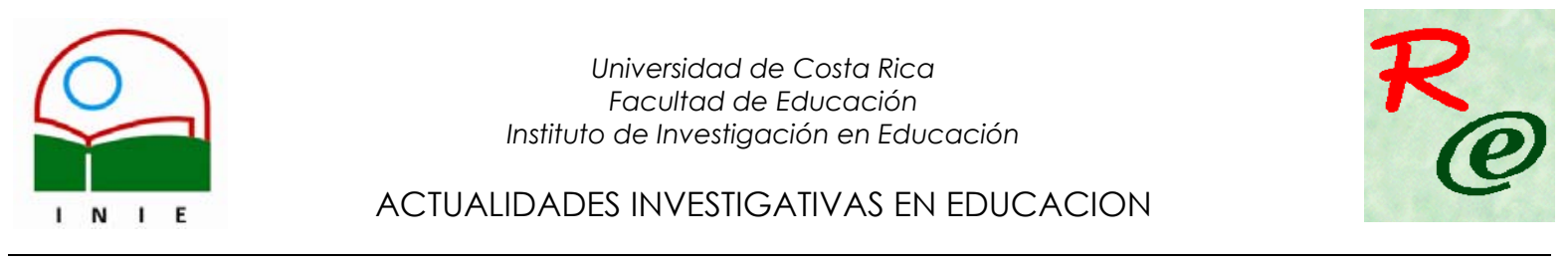

\title{
LA MOTIVACIÓN EN LOS ESTUDIANTES UNIVERSITARIOS
}

\section{Ana Polanco Hernández ${ }^{1}$}

\begin{abstract}
Resumen: Este artículo presenta el complejo tema de la motivación en el estudiante universitario. Se plantean los componentes y las características del proceso emotivo, así como la motivación intrínsica y la extrínseca. Estos factores pueden favorecer la motivación en los estudiantes, como lo son, enlazar los conocimientos nuevos con los ya adquiridos; ofrecer un material significativo; organizar las experiencias de aprendizaje, en función de los estudiantes y no del docente; plantear en cada clase, expectativas que provoquen interés para la siguiente lección; organizar el contenido, de tal forma, que promueva el interés en los alumnos.
\end{abstract}

Palabras clave: MOTIVACIÓN/ EDUCACIÓN SUPERIOR/ ESTUDIANTES UNIVERSITARIOS/ UNIVERSIDAD APRENDIZAJE/

Abstract: This article deals with the university-level student's motivation and its complexity. The components and characteristics of the motivational process, and these of the intrinsic and extrinsic motivation, are stated here. The factors that help to motivate students are: linking up the new knowledge with the previously adquired concepts; offering meaningful materials; organizing the learning experiences in function of the students instead of in function of the teachers; giving the students for the fallowing lessons to inspire a greater interest; and finally, preparing the courses content to improve the student's interest towards the lesson.

Keywords: MOTIVATION/ HIGHER EDUCATION/ UNIVERSITY-LEVEL STUDENTS/ LEARNING IN THE UNIVERSITYI

\section{Introducción}

La palabra "motivación", ha sufrido un cambio progresivo, desde el punto de vista conductista, hasta las orientaciones cognitivas actuales. Su polémica gira en torno a los factores que pueden influir y la diferencia entre el interés que se presenta por una tarea. Efectivamente, es obvio que las atribuciones del éxito o fracaso, son factores que determinan la motivación de los educandos. Sin embargo, su estudio es complicado, porque tiene a su disposición, muchas teorías para analizar, e investigaciones y tesis controversiales sobre el tema.

1 Master en Planificación Curricular, Licenciada en Educación
Preescolar, Bachiller en Educación Preescolar, todos los títulos de la
Universidad de Costa Rica. Profesora de la Escuela Formación
Docente, Sección de Preescolar. Coordinadora Proyecto de Acción
Social "Apoyo Docente a Instituciones Públicas de Educación Inicial".

Correo electrónico: apolanco@costarricense.cr

Artículo recibido: 12 de junio, 2005

Aprobado: 22 de agosto, 2005 
La pregunta, si es necesaria la motivación para que se lleve a cabo el aprendizaje, ha ocasionado diversas controversias, dado que las posiciones varían desde la afirmación de que ningún aprendizaje se realizará, si no existe motivación, hasta la negación completa de la motivación, como variable importante para que se realice este proceso Ausubel (1981). Esto porque se presenta una considerable cantidad de aprendizajes que no son impulsados por la motivación, pues ocurren de manera incidental y sin una intención explícita. Sin embargo, al referirse a la motivación para el aprendizaje, se da una relación recíproca, pues se dan situaciones de aprendizaje promovidas por el docente sin que necesariamente surjan con anterioridad los intereses y las motivaciones de parte de los estudiantes, pero que dichos aprendizajes no pueden postergarse.

Es un hecho que la motivación influye en el aprendizaje, hasta el punto de llegar a ser uno de los principales objetivos de los profesores: motivar a sus estudiantes. Sin embargo, la falta de motivación es una de las causas importantes que se debe valorar en el fracaso de los estudiantes, especialmente cuando se fundamenta en la distancia establecida entre los actuantes del proceso. Así, se puede observar que la relación profesor estudiante, en el contexto universitario, se presenta, generalmente, en forma lejana, y la impersonalidad que se concibe no permite centralizar la atención en el sujeto que aprende, sino más bien la formación universitaria tiende a identificar el punto fundamental del aprendizaje en el sistema, entre el profesor y el contenido. Este modelo se introduce en una sociedad, cuya finalidad inmersa en un proceso globalizante, se ubica frente a intereses de carácter cuantitativo y sistemas técnicos de manejo de información, lo que desdeña el sistema de transmisión de conocimientos de forma humanizante, ya que deja de lado al sujeto que aprende.

Santos (1990), define la motivación como "el grado en que los alumnos se esfuerzan para conseguir metas académicas que perciben como útiles y significativas". Desde el punto de vista del docente, significa "motivar al estudiante a hacer algo, por medio de la promoción y sensibilización" (Campanario, 2002) "Motivar supone predisponer al estudiante a participar activamente en los trabajos en el aula. El propósito de la motivación consiste en despertar el interés y dirigir los esfuerzos para alcanzar metas definidas".

Desde una perspectiva histórica, Abarca (1995) se refiere a la motivación como un fenómeno integrado por varios componentes, los cuales aparecen y desaparecen de acuerdo con las circunstancias determinadas por los fenómenos sociales, culturales y económicos y, por ende, debe tener un tratamiento particular para cada uno de los sujetos. 
Definitivamente, la interacción de factores hace que la motivación en contextos académicos resulte una tarea sumamente compleja, ya que cada uno de estos factores cumple una función específica. Según Alonso Tapia, (1991) las metas que persiguen los alumnos pueden clasificarse, con base en varias categorías que no son completamente excluyentes:

a. Metas relacionadas con la tarea: la motivación por alcanzar una meta hace que el individuo sea más persistente, aprenda más eficazmente y tienda a llegar a conclusiones antes que otros estudiantes El logro de esta meta se da cuando el estudiante logra tomar conciencia de que la tarea ha sido superada y que ha logrado un mejoramiento en una de las áreas que le interesa. Así Ausubel (1981, p. 430) plantea con respecto a esta tarea, que el motivo por adquirir un conocimiento en particular, es intrínseco a la tarea, consiste sencillamente en la necesidad de saber y por lo tanto el lograr obtener este conocimiento, afirma completamente la tarea, ya que es capaz de satisfacer el motivo subyacente. De esta manera, al ubicarse en un contexto universitario, la posibilidad de aplicar conocimientos, desarrollar habilidades y elaborar nuevos esquemas de conocimiento, puede provocar un incremento en el nivel de motivación.

b. Metas relacionadas con el "ego": Al relacionarse el estudiante con otros, se tiene percepciones del mundo que se forman, desde la historia personal de cada uno de los individuos, el sentirse superior al otro o, bien, demostrarles a los demás, sus capacidades y destrezas propias, permite lograr una reconciliación con el "ego" y la satisfacción personal de éxito. Ausubel (1981) le llama a esta meta "mejoramiento del yo" porque se refiere al aprovechamiento, como fuente de estatus ganado, a saber, el lugar que gana en proporción con su nivel de aprovechamiento o de competencia. Esta meta es la que se presenta, con más fuerza, en la mayoría de las culturas, especialmente, en la occidental. Esta motivación produce mayor persistencia, más éxito en la solución de problemas y mayor aprovechamiento académico a corto y largo plazo.

c. Metas relacionadas con la valoración social: cuando el individuo se enfrenta a una sociedad, se desarrolla la necesidad de aceptación y reconocimiento de las virtudes y aprobación, tanto de padres como maestros y compañeros. La experiencia de evitar el rechazo es un elemento de motivación en el nivel de grupo. Ausubel (1981) propone con respecto a esta meta, que ésta no se refiere al aprovechamiento académico, como fuente de estatus primario, si no que se orienta hacia el aprovechamiento que le asegure la aprobación de una persona o grupo. Efectivamente, en la sociedad estudiantil, se destacan características particulares que el estudiante se esfuerza por ofrecer ante los demás y, de esta manera, obtener una recompensa. 
d. Metas relacionadas con la consecución de recompensas externas: este factor está vinculado con el acceso a una posición social, un estatus económico, u otras posibilidades de recompensas externas, como becas, premios, certificados, entre otros.

Otro aspecto importante que se relaciona con la motivación es el concepto de "necesidad" (Abarca 1995) vinculado con el aprendizaje, al considerar que la necesidad del individuo es un factor primordial para propiciar la motivación, cada quien desde su percepción histórica se refiere a una motivación particular que nace de la necesidad individual, vista como fuerza pujante desde su propio punto de vista.

La motivación puede surgir por medio de dos procesos: intrínseco y extrínseco. Cuando un estudiante tiene una motivación intrínseca, está motivado por la vivencia del proceso, más que por los logros o resultados del mismo, lo que provoca que estudie por el interés que le genera la materia. En este caso, la autorregulación cognitiva, la independencia y autodeterminación son cualidades evidentes del sujeto. Así según Raffini (1998, p. 13), la motivación intrínsica es elegir realizar un trabajo por la simple satisfacción de hacerlo, sin nada que nos obligue o apremie, Esto es lo que nos motiva a hacer algo, cuando nada exterior nos empuja a hacerlo.

Lo opuesto busca obtener una recompensa, lo que permite visualizar el logro como una experiencia que podría acarrear frustración y desencanto hacia una tarea, materia, persona o área específica del conocimiento que no le genera premios.

Por el contrario Campanario (2002) comenta que la motivación extrínseca se produce, cuando el estímulo no guarda relación directa con la materia desarrollada, o cuando el motivo para estudiar, es solamente la necesidad de aprobar el curso.

Así se pueden comentar los componentes del proceso motivacional y sus características, según Abarca (1995), a saber las necesidades, los intereses y los motivos. Con respecto a las necesidades, estas se definen como (Abarca 1995:8) "la fuerza que impulsa a los hombres y a las mujeres a actuar, a moverse y a encontrar los medios para satisfacer sus demandas". Por esto es importante que el estudiante sienta la necesidad de descubrir para satisfacer su carencia, si no es así es difícil que se dé un aprendizaje. El educador debe crear estrategias que faciliten la necesidad en el alumno por alcanzar un determinado aprendizaje, ya que si el objeto de estudio es agradable e interesante para él, esto hará que aumente su necesidad, creando una fuerza interna consistente y fuerte en la que el individuo busca su propia gratificación personal sin necesidad de exámenes. Pero lamentablemente, 
esta situación no se da a menudo en los estudiantes universitarios. Por el contrario, el profesor utiliza controles como las tareas y pruebas para que se produzca el aprendizaje.

Abarca (1995) plantea también las necesidades fisiológicas, las sociales y las de logro, a continuación se comentarán brevemente sus aspectos relevantes. Así las necesidades fisiológicas, se relacionan con la necesidad del ser humano de cubrir aspectos básicos como el alimento, abrigo, saciar la sed. Es importante que estas necesidades se satisfagan en forma equilibrada para que se facilite la construcción de conocimientos y de aprendizaje.

Con respecto a las necesidades sociales o secundarias, estas nacen a partir de las relaciones con otros seres humanos, con la cultura, la familia, las personas que poseen distintos valores y costumbres. Por consiguiente esta experiencia social es única y diferente en cada individuo. (Predvechni, 1993, p. 82 citado por Abarca 1995, p. 13) las define como "La satisfacción de las necesidades elementales es la base, el trampolín para el surgimiento de las necesidades secundaria indispensables, tanto materiales como espirituales (conocimiento, educación, instrucción), así como para el de las necesidades no indispensables (fumar, etc)"

Por último, la necesidad de de logro que para Abarca (1995, p. 17) "es el requisito de alcanzar metas, de superarse, de descollar, de obtener altos niveles de desarrollo personal" Todas las personas tienen esta necesidad en mayor o menor grado, lo cual determina la perseverancia, la calidad, los riesgos que se toman y la consistencia para hacer frente a sus retos y objetivos.

Con respecto al segundo componente del proceso motivacional "los intereses" significan deseos de conocer y aprender, de practicar una disciplina o arte. Por lo tanto, cada interés presenta un sentido emocional para cada individuo y pueden variar con el tiempo o las circunstancias.

Los intereses según Petrovski 1980, citado por Abarca (1995, p. 21), pueden distinguirse por: el contenido, la finalidad, la amplitud, la constancia y el género.

Por último el tercer componente se denomina "los motivos" Los cuales están muy relacionados con las necesidades y se definen como "... móviles para la actividad relacionados con la satisfacción de determinadas necesidades" (Abarca 1995, p. 25). No se puede juzgar a simple vista el tipo de necesidades que aparenta un motivar a un ser humano, esto por cuanto varias personas pueden estar realizando una misma actividad por motivos diferentes.(sociales, fisiológicas, de logro). Los intereses como parte de las circunstancias históricas y los motivos; como móviles del proceso, intervienen como parte de un momento particular determinado por la percepción histórica de su propio mundo. 
En el caso de la población estudiantil en la Universidad de Costa Rica, se deben tener considerar algunos factores específicos, como lo son: la manera de ingreso a cada carrera; oportunidades de elección vocacional; reconocimiento social de las diferentes carreras, mercado laboral, entre otras.

Con respecto a formas de ingreso a carrera, éstas pueden variar de acuerdo con cada centro de educación superior. Así en el caso de la Universidad de Costa Rica, la posibilidad para el estudiante, de inscribirse en la carrera que desea, depende, en la mayoría de los casos, del puntaje obtenido en la prueba de aptitud académica, o bien una combinación de esta prueba con habilidades que se requiere para la carrera, como por ejemplo Arquitectura o Bellas Artes. En lo que se refiere a las oportunidades de elección vocacional, a menudo, el estudiante no ha tenido la oportunidad de conocer la gama de posibles profesiones que se le ofrecen, aunado al autoconocimiento de sus habilidades y deseos profesionales. El reconocimiento social juega un papel importante en la elección, pues hay quienes deciden seguir estudios de una carrera que no les satisface a nivel personal, pero que la presión social y familiar los induce a una elección profesional no acertada.

Estos son algunos elementos que provocan que, en ocasiones, los estudiantes no ingresen a la carrera que más les interesa y este es un factor que puede llegar a afectar

La verdadera motivación del estudiante universitario, es aprender en un ambiente de universalidad del conocimiento, pues cada aprendizaje logrado le permite diversificarse en la carrera escogida y le ofrece un escalón más en la meta hacia su logro académico. Este concepto de universalidad en la educación universitaria, está adherido a la influencia del quehacer, aunado a las nuevas tendencias globalizantes y de competencia del mercado y cumple una función importante en el discurso pedagógico, el cual podría reforzar o influir en el grado de motivación de los estudiantes, ya que una persona motivada es aquella que muestra persistencia en una carrera o una actividad.

Por otra parte, en ocasiones resulta más simple achacar los problemas de la motivación del aprendizaje, a factores propios de los alumnos, que a factores relacionados con la actuación del docente. En este punto, se debe enfatizar la importancia de la función docente y las estrategias de enseñanza en la motivación de los estudiantes, tomando en cuenta los factores intrínsecos y extrínsecos de la motivación, como puntos de partida para contemplar las necesidades individuales y la atención de los estudiantes, como seres humanos con una historia previa que determina sus enfoques particulares en cuanto a la motivación.

La posibilidad de motivar a los alumnos, desde el punto de vista docente, no implica rebajar el nivel de la enseñanza, se debería hacer más que divertir e interesar al alumno que se 
desea mantener motivado. Hay tres factores a nivel del aula que es posible utilizar como orientaciones motivacionales: la estructura de la tarea, el mecanismo de recompensa y la forma de ejercer la autoridad.

La organización de la tarea se da como parte de la interacción de las tres estrategias mencionadas anteriormente, para el alcance de formas de presentación: individualistas, cooperativa y competitiva. En la función individualista, las tareas son independientes y la recompensa en una calificación en función de su actuación y sus resultados. En la organización cooperativa, trabajan en grupos pequeños y la calificación depende del aporte personal y el resultado de los restantes miembros del grupo. En la competitiva, los estudiantes trabajan sin relación entre sí y reciben calificaciones que dependen negativamente de los resultados que obtengan los demás. Esta modalidad es usualmente utilizada por los profesores de enseñanza secundaria y universitaria; la cual, algunas veces, es individualista y se convierte en competitiva.

Es real que el trabajo en grupo produce más satisfacción a nivel de motivación en los estudiantes; no obstante, debe tenerse presente que este trabajo debe realizarse en equipo , y no se trata de que cada miembro contribuya, individualmente, con una parte del mismo para luego unirlas y formar "un todo". Por el contrario. El objetivo plantea que, en conjunto, los individuos investiguen, discutan, comenten el trabajo por ejecutar, lo que dará como resultado, un aporte más homogénea de parte de cada alumno.

En la bibliografía educativa se encuentran investigaciones que demuestran las ventajas motivacionales del trabajo en grupo cooperativo, en los niveles de enseñanza secundaria y universitaria (Johnson y Jonson, 1985). Pero más allá de esto, también debe prestarse atención a las interacciones que se dan dentro del grupo y de los grupos entre si (Windschit, citado por Campanario 2002). Este tipo de estrategia de enseñanza grupal fomenta una motivación intrínseca más sana y consigue concentrar la atención en los procesos, más que en los resultados en general y fomenta el interés en la tarea. Obviamente, las perspectivas de éxito son mayores en un entorno en el que la ayuda de los demás, es un factor con el que se puede contar en principio. Este tipo de estrategia, fomenta actitudes sociables entre los alumnos; promueve la mutua cooperación y la aceptación de ayuda; e intensifica la tolerancia. En oposición a una estructura competitiva, donde cualquier ayuda de un compañero, podría convertirse en su propio perjuicio.

Paralela a la estrategia de trabajo grupal que se da por medio de una motivación intrínseca y fundamentada en los procesos, hay múltiples factores que pueden influir en el grado de motivación de los estudiantes. Por ejemplo, la manera de presentar los conocimientos 
nuevos y comentar las declaraciones inciertas, la enseñanza de algo sorprendente, el planteamiento de problemas que atraiga a los estudiantes a resolverlos, variación de las tareas, el método de enseñanza, entre otros.

Aunque estos factores podrían influir en la motivación de los estudiantes, dadas las particularidades de cada uno, un método podría ser motivador para un grupo de estudiantes y no serlo para otros. Así, se podría decir que los estudiantes con diferentes características motivacionales, presentan necesidades diferentes en relación con el método de enseñanza. Pero es fundamental entender que la motivación es un proceso cambiante y que se llega a desarrollar en el contacto con el medio y con factores, como la clase social, género, raza, entre otros, los cuales son evidentemente elementos a considerar en la elaboración de una estrategia motivacional.

Las necesidades, motivos e intereses se modifican a partir del individuo y del momento histórico, de modo que el conocimiento del grupo o la población, en sus particularidades más individuales es un elemento indispensable de la estrategia.

Asimismo, la flexibilidad y atención ante el cambio que debe darse por parte del educador y la oportunidad de tener experiencias significativas que produzcan interés, son elementos propios del proceso.

A continuación se presentan algunos factores que pueden favorecer la motivación en los estudiantes (Abarca 1995):

\section{- Enlace entre los conocimientos previos y los nuevos}

No es conveniente partir de la idea de que los estudiantes, antes de ingresar al aula, no tienen ningún conocimiento sobre el tema que se vaya a tratar. Sin embargo, en ocasiones, los profesores conciben al estudiante como "una tabla rasa", lo cual es una visión pedagógicamente objetivista y contraria a una posición constructivista, según lo plantea Abarca (1995, p. 121).

Para Ausubel citado por Novak 1982, p. 71) el enlace entre los conocimientos previos y los nuevos, contribuye a lograr lo que él denomina "Aprendizaje significativo", al mencionar que este es un proceso por el que se relaciona, nueva información con algún aspecto ya existente en la estructura cognitiva del alumno y que es relevante para el aprendizaje que intenta aprender. Al darse este proceso, los conjuntos de células que intervienen almacenando información en el aprendizaje, sufren cambios adicionales y, es probable, que formen sinapsis o algún tipo de unión funcional con neuronas nuevas. Para lograr esto, el 
profesor debe acercarse a sus alumnos y conocer sus intereses, necesidades, conocimientos previos, partir de lo conocido y lograr provocar un aprendizaje significativo, que perdure.

\section{- El significado del material para el alumno:}

Este aspecto es relevante, dado que utilizar diferente material en el aula, puede provocar en el alumno, mayor motivación e interés por el tema que se está tratando. El material debe provocar ilusión, reto cognitivo y deseo de conocer. Por lo tanto, no pueden ser aburridos, rutinarios o demasiado simples. Por el contrario los materiales con alto grado de complejidad, no son necesariamente desmotivantes ni dejan de ser significativos. Una prueba de ello son algunos juegos computarizados complejos que atraen la atención de los jóvenes universitarios.

Por otro lado es responsabilidad del profesor el conocer y utilizar los materiales tecnológicos que existen en la actualidad y que tiene a disposición para impartir lecciones, dado que no puede obviar que los estudiantes de hoy día viven en un medio tecnológico mucho más rico, que les brindan posibilidades distintas a las que se ofrecían en el siglo pasado. Así una forma de motivar a los estudiantes, es utilizar estrategias modernas.

Por otro lado, al elegir un material debe verificarse que posea poder explicativo, que vaya de lo simple a lo complejo, de lo más amplio a lo más específico, de lo general a lo particular, hasta llegar al conocimiento de las especialidades

\section{- La organización de la experiencia de aprendizaje.}

La organización de las experiencias de aprendizaje se dan de acuerdo con las creencias que tiene el educador, de cómo es que sus estudiantes aprenden la materia de su curso. Por ello Abarca (1995:128) plantea que lo importante entonces es, saber cómo el docente concibe el proceso de construcción del conocimiento de sus alumnos. Por este motivo, debe darse una planificación en función de los alumnos y no de él.

Se propone hacer un diagnóstico de los conocimientos previos de los estudiantes, sus habilidades y estilos de aprendizaje.

Es importante realizar una distribución de los contenidos que deben tratarse durante el semestre, y en cada sesión. No obstante, esta propuesta debe ser flexible para atender las necesidades de los alumnos. 


\section{- El grado de expectativa o reto}

Es importante que el estudiante perciba cierta expectativa en cada una de las clases; esto lo motiva a interesarse por la siguiente lección y mantener su motivación. Una de estrategia recomendable por seguir, es promover que el alumno sea un protagonista en el aula y se apropie del conocimiento, en donde su posición no se reduzca a escuchar y repetir la materia.

Es recomendable que el profesor considere algunos aspectos, como pueden ser:

- Hacer sentirse a los alumnos responsables por su proceso de aprendizaje.

- Dar a conocer a los estudiantes el propósito de los diferentes trabajos que se le solicita que realicen.

- Fomentar la interacción del alumno con el objeto de conocimiento, que se sienta capaz de trabajar con un determinado método, guía de trabajo, texto; de esta forma, descubre sus limitaciones y habilidades

- $\quad$ Es responsabilidad del profesor al realizar su planificación didáctica, ser creativo, crear expectativas y promover la motivación en sus estudiantes.

- $\quad$ Por último pero no por ello menos importante, está la claridad que se debe tener de los objetivos que se pretende que los estudiantes logren alcanzar.

\section{- Características del objetivo de estudio}

Se entiende por objeto de estudio cualquier proceso, conocimiento, método o información que el estudiante debe llegar a conocer.

El docente debe tener claridad de las características de su asignatura; las habilidades cognitivas que deben poseer los estudiantes para dar significado a la materia; así como una organización del contenido que promueva el interés.

El objeto de estudio nace en un contexto y en un tiempo; por ello constantemente, puede cambiar, y surge la necesidad de estar en continua actualización, si el estudiante se conscientiza de esto, estará motivado a buscar nueva información, y no limitarse a lo proporcionado en clase.

Es esencial que el educador tenga un balance en la cantidad de contenidos que ofrece en su curso para no saturar al estudiante, ni tampoco subestimarlo en su capacidad de aprendizaje. 


\section{Conclusión}

Para mantener el interés del educando, se debe aprovechar la energía natural del alumno para sentirse capaz y orientado hacia el logro de metas. Es necesario que realice de manera voluntaria, lo que se espera que haga; y que desarrolle sus aptitudes para que alcance la meta deseada. Así, cuanto más capaz se sienta un alumno de desarrollar una actividad, más motivado estará para persistir en ella, a su vez, le dará la sensación de éxito o de mejoría y le ayudará a mantenerse motivado.

El docente debe recordar que el alumno, como ser humano, siente la necesidad de proteger su autoestima, por lo que es importante buscar experiencias que generen sentimientos de orgullo y de satisfacción. Además se debe ofrecer comentarios positivos para ayudarle a que preste atención a sus características individuales y a asumir la responsabilidad de sus propias acciones.

Por último el docente debe tener presente que el alumno necesita la posibilidad de explorar opciones; recibir comentarios positivos de sus fortalezas y debilidades.

Así, la posibilidad de coadyuvar los elementos de estudio intrínsecos en el análisis del éxito o fracaso escolar, podrían dar un proceso eficiente cuando la ubicación de los adecuados intereses, necesidades, componentes motivaciones, momento histórico adecuado y recursos, en general, se complementen en un proceso totalmente subjetivo ya que el individuo mismo es el actuante de este fenómeno interactivo, social y fisiológico; en el cual la concordancia adecuada, del elemento motivacional puede ser interferido por factores totalmente externos a la realidad educativa. 


\section{REFERENCIAS}

Abarca, Sonia. (1995). Psicología de la motivación. San José, C.R.: Editorial Universidad Estatal a Distancia.

Ausubel, David. (1981). Psicología educativa. Un punto de vista cognoscitivo. México: Editorial Trillas.

Barquero, Ricardo. (1997). Vigotsky y el aprendizaje escolar. Buenos Aires, Argentina: Editorial Aique.

Campanario, Juan Miguel. (2002). ¿Cómo influye la motivación en el aprendizaje de las ciencias? Recuperado el 15 de marzo de 2005 en http://www2.uah.es/imc/webens/127.html

Criterios pedagógicos para el diseño de materiales educativos. (2002). Recuperado el 15 de marzo de 2005 en http://investigacion.ilce.edu.mx/dice/diplomado/html.html

Cómo puedo organizar las actividades de clase para motivar a los alumnos. (2002). Recuperado el 15 de marzo de 2005 en http://www2.uah.es/jmc/webens/portada.html

Fabret, Jaime. Enfoque de los contenidos del curso "Estrategias motivacionales". 15 de marzo de 2005 en http://www.ma1,upc.es/recerca/reportstre/001fabregat.doc

Fabregat Fillet, Jaime (2000) Programa de Cooperación Interuniversitaria. Ministerio de Asuntos Exteriores Agencia Española de Cooperación Internacional [En red] www.ma1.upc.edu/recerca/reportstre/00/rep0001fabregat.doc

Garcia Tapia Francisco. Taller de Estrategias Didácticas para la Enseñanza de la Biología. 15 de marzo de 2005, en http://redescolar.ilce.edu.mx/redescolar/biblioteca/articulo/art.tm-dd

Johnson y Johnson. (1985). Motivational processes in cooperative, competitive and individualistic learning situations. Research on Motivation in Education, 2, Academic Press: New York - EE.UU.

Maldonado, Gonzalo. El aprendizaje significativo de David Ausubel. Recuperado el 2 de marzo de 2005 en $\underline{\text { www.apuntesuniversitarios.com/EvaluacionAprendizaje/evaluapre.html }}$

Maldonado Osorio, Gonzalo. Resumen Analítico. Recuperado el 15 de marzo de 2005 en www.apuntesuniversitarios.com/EvaluacionAprendizaje/evaluapre.html

Mollier, María Angélica. El fracaso escolar en la última década del siglo XX. Recuperado el 15 de marzo de 2005 en http://www.unrc.edu.ar/publicar/cde/eh4.html

¿Motivar a los alumnos implica necesariamente rebajar el nivel de la enseñanza (2002) [En red] http://www2.uah.es/jmc/webens/portada.html 
Novak, Joseph. (1982). Teoría y práctica de la educación. Madrid España: Editorial Alianza.

Novak, J. y Gowin B. Aprendiendo a Aprender. Recuperado el 2 de marzo de 2005 en www.apuntesuniversitarios.com/EvaluacionAprendizaje/evaluapre.html

Raffini, James. (1998). 150 Maneras de incrementar la motivación en la clase. Argentina: Editorial Troquel.

Ramo García, Arturo. (1999). Motivación del aprendizaje. Recuperado el 15 de marzo de 2005 en http://www.educa.aragob.es/aplicadi/didac/dida38.htm.

Por qué no es lo mismo organizar el trabajo en grupos cooperativos que pone a los alumnos a trabajar en equipo? Recuperado el 15 de marzo de 2005 en http://www2.uah.es/jmc/webens/portada.html

¿Por qué el trabajo mediante grupos cooperativos tiene un efecto tan positivo en la motivación y el aprendizaje? Recuperado el 15 de marzo de 2005 en http://www2.uah.es/jmc/webens/portada.html

¿Podemos analizar algún ejemplo concreto de estrategia de motivación? Recuperado el 15 de marzo de 2005 en http://www2.uah.es/jmc/webens/portada.html

¿Por qué no se debe confundir interés con motivación? Recuperado el 15 de marzo de 2005 en http://www2.uah.es/jmc/webens/portada.html

¿Qué tienen que ver las metas del trabajo escolar y las atribuciones de éxitos o fracasos con la motivación de los alumnos? Recuperado el15 de marzo de 2005 en http://www2.uah.es/jmc/webens/psicolog.html\#38 